Psychological Medicine, 1982, 12, 13-15

Printed in Great Britain

\title{
EDITORIAL
}

\section{Motor disorders in schizophrenia ${ }^{1}$}

The article by Manschreck et al. published in this volume of Psychological Medicine (pp. 73-84) on 'Disturbed voluntary motor activity in schizophrenic disorder' is of considerable interest. The authors claim that 'Disturbance in voluntary motor activity...not attributable to drug effect or known neurological disorder ... occurs in virtually all cases of conservatively defined schizophrenia'. The authors consider this finding compatible with the 'attention-deficit' hypothesis of schizophrenia, which predicts that motor and thinking disturbances should coincide in this disorder. Such a claim deserves close scrutiny and my purpose here is to examine the methods employed to detect this so-called disturbance of motor activity. In particular, I wish to draw attention to $(a)$ the uncertainty of claims that the sort of florid motor disturbances now associated with organic brain disease existed in patients with schizophrenia prior to the neuroleptic era; $(b)$ the subtle role of neuroleptic drugs in causing disturbances of voluntary motor activity; and $(c)$ the difficulties inherent in using apparently validated rating scales as objective methods of assessing motor disorder.

Manschreck et al. draw attention to the long history of descriptions of abnormal motor features in schizophrenia, even before the advent of neuroleptic drugs in the 1950s. Prior to this time, schizophrenia was contaminated by the aftermath of the epidemic of encephalitis lethargica. Encephalitis lethargica could produce both a psychotic illness, with features easily confused with those of schizophrenia, and a wide range of movement disorders. The description of 'schizophrenia' by Bleuler in 1911 preceded the appearance of the epidemic of encephalitis lethargica by only a few years, and sporadic cases and small outbreaks of encephalitis lethargica appear to have occurred even prior to 1916/17; sporadic cases occasionally can be seen today. Reading Kahlbaum's (1874) monograph introducing the concept of catatonia, one is struck by the similarity of the description of some of his patients to those suffering from post-encephalitic Parkinsonism. Benjamin L., the first case he described, fell ill, silent and disinterested, with choreiform facial ' tics' and spastic jerking movements of the extremities, and with a still immobile posture, as well as episodes in which he directed his gaze partially upwards for hours on end. Syphilis, which too can cause a wide range of movement disorders, also was rife at that time. Indeed, Kahlbaum's second patient with catatonia, Julius G., was known to have suffered from syphilis. Kraepelin (1913) acknowledged that 'considerable difficulties, which at present cannot always with certainty be overcome, may arise, when there is a question whether certain acute hallucinatory states of excitement in patients demonstrably syphilitic are to be regarded as the expression of syphilis or of dementia praecox'.

Against this background of the prevalence of organic diseases such as encephalitis lethargica and syphilis, it is exceedingly difficult to assess whether the patients with true schizophrenia before the era of drug therapy really did exhibit abnormal movements of the sort now seen commonly in neuroleptic-treated individuals. The plethora of motor disorders described in the pre-neuroleptic period undoubtedly was seen most commonly among those with catatonic schizophrenia. It may not be coincidence that this form of the illness has now virtually disappeared, at the same time that penicillin has all but abolished tertiary neuro-syphilis and encephalitis is now a rarity. In reviewing this problem (Marsden et al. 1975), we concluded that 'it is likely that many choreo-athetotic signs described in the older literature occurred as a result of organic neurological disease. More recent studies indicate that true chorea or athetosis is extremely rare in chronic psychiatric populations and, when present, is nearly always associated with organic neurologic disease... Indeed, the presence of typical chorea or dystonia in a psychotic patient should raise the possibility that the illness is symptomatic of an organic extrapyramidal disorder.'

\footnotetext{
1 Address for correspondence: Professor C. D. Marsden, Department of Neurology, Institute of Psychiatry, De Crespigny Purk, Denmark Hill, London SE5 8AF.
} 
Manschreck et al. however, concentrate in their paper not on the gross abnormal movements characteristic of neurological disease, but on more subtle abnormalities of motor activity. In particular, they looked for those features of spontaneous motor action characteristic of schizophrenia (such as motor blocking, stereotypies and mannerisms, and general clumsiness), an abnormal performance on a range of motor tasks undertaken (such as eye opening and closure, stance and gait, tests of arm coordination), and the capacity to execute sequential motor acts (such as the fist-edge-palm test). They found abnormal spontaneous and elicited motor activity in nearly all of a group of 37 schizophrenic patients: 25 of the 37 subjects exhibited some abnormality of spontaneous movement, and 32 of elicited movements. Were these motor disorders part of the schizophrenia, or were they due to neuroleptic drug intake?

Manschreck et al. marshal much evidence which, in their view, excludes the influence of neuroleptic drugs. They note that 5 schizophrenic patients were believed not to have taken neuroleptics in the previous year, and 2 apparently had never taken such drugs. Yet these untreated schizophrenics had more motor abnormality than drug-treated patients. In addition, more motor abnormality was found in the schizophrenic patients as a whole than in another population of 16 patients with affective disorders. Since many of the spontaneous motor abnormalities recorded are known to be characteristic of active schizophrenia, and since motor changes such as blocking and stereotypy interfere with the elicited motor tasks studied, these findings are not surprising. What is open to question is the belief that stopping neurroleptic drugs for a year removed their effects on the motor system.

Clinical experience shows that this is manifestly not true. Drug-induced parkinsonism may take as long as 18 months to show signs of improvement, and as long as 5 years to disappear. Drug withdrawal commonly exacerbates neuroleptic-induced tardive dyskinesias, which may appear for the first time once the offending drug is stopped. The rate of remission of neuroleptic-induced tardive dyskinesias also is related to the period of drug withdrawal, such that few would now accept a period of less that 5 years free of drug before considering tardive dyskinesia 'permanent'. To compare a group of patients on neuroleptic drugs with another group in whom the drugs have recently been stopped may give rise to misleading conclusions. The only real control for drug effects is a population never exposed to neuroleptics, but this is hard to find. Even in hospitals or wards believed not to have used neuroleptic drugs, there may be covert administration of these agents unbeknown to the medical staff.

Manschreck et al. also tried to control for the effect of neuroleptic drugs by examining the incidence of motor disturbances with that of frank extrapyramidal side-effects of neuroleptics, as judged by two rating scales. The two scales utilized were the Abnormal Involuntary Movement Scale (AIMS), and the Targeting of Abnormal Kinetic Effects (TAKE) scale. AIMS is believed to rate the severity of tardive dyskinesias, while TAKE assesses extrapyramidal effects and akathisia. $37 \%$ of the sample of schizophrenic patients studied by Manschreck et al. exhibited such complications of neuroleptic drug treatment as assessed by these two rating scales. Disturbance of 'motor activity' did not correlate with positive scores of AIMS and TAKE; Manschreck et al. believed that this provided added evidence to exclude drug effects as responsible for the disturbances in voluntary performance they found. Both rating scales, however, are open to criticism. AIMS, although showing high inter-rater reliability, does not clearly distinguish between normal oro-facial behaviour of the elderly, edentulous subject with a drug-induced dry mouth, and a true unequivocal tardive dyskinesia. A cut-off point of about 2 or more on the AIMS scale is now taken to indicate the presence of real tardive dyskinesia, but the division is not clear. TAKE also has good inter-rater reliability, but does not allow for the damaging effect of mild neuroleptic-induced disturbances of motor activity. The subtlest sign of neuroleptic-induced extrapyramidal action is slight akinesia, which is evident only as an interference with voluntary action. There may be no obvious loss or poverty of movement, no rigidity and no tremor, but only a subtle disturbance of normal motor skill. Of course, this is just what is seen in idiopathic Parkinson's disease, in which an inability to execute skilled and sequential motor acts is often the earliest and subtlest sign of the illness. TAKE is not sensitive to such changes, but the tests utilized by Manschreck et al. to study elicited motor 
behaviour involved just those skills compromised by both Parkinson's disease and early neurolepticinduced Parkinsonism. In other words, their tests of elicited motor activity could not have been designed better to pick out extrapyramidal side-effects of neuroleptic drugs. In summary, AIMS is too sensitive to be sure that it is detecting tardive dyskinesia, while TAKE is too insensitive to pick up all the parkinsonian side-effects of neuroleptics. Such deficiencies in the instruments employed make it extremely unlikely that Manschreck et al. would have found any close correlation between neuroleptic-induced motor disability and the movement disorder that they studied.

What can be concluded from all this? First, any study purporting to investigate motor disorders in schizophrenia must distinguish between $(a)$ those disturbances of motor ability known to be characteristic of the illness, and the effects of these motor abnormalities and altered volition upon both spontaneous and elicited motor skills; and $(b)$ extrapyramidal movement disorders induced either by neuroleptic drugs or organic disease. The rating scales presently available for extrapyramidal side-effects are inadequate either to exclude or to establish with certainty the presence or absence of such phenomena. Unfortunately, drug histories are notoriously unreliable, and it is now clear that a very long period must elapse between stopping neuroleptic treatment and the time at which all its effects upon motor performance disappear. The only convincing way to examine motor changes in schizophrenia is to study, prospectively, patients who are pharmacologically 'virgin'. To obtain such a population would demand the closest collaboration with primary physicians alerted to refer possible schizophrenic patients to specialists interested in this problem before starting drug treatment. Against this background of considerable difficulty surrounding the whole of this topic, Manschreck et al. must be congratulated in teasing out the fact that patients with schizophrenia do have some abnormalities of volitional motor activity, as highlighted in the original descriptions of the disorder.

C. D. MARSDEN

\section{REFERENCES}

Bleuler, E. P. (1911). Dementia Praecox or the Group of Schizophrenias (English transl. J. Zinkin, 1950). International Universities Press: New York.

Kahlbaum, K. L. (1874). Die Katatonie oder das Spannungsirresein (English transl. Y. Levij and T. Pridan, 1973). Johns Hopkins University Press: Baltimore.

Kraepelin, E. (1913). Dementia praecox and paraphrenia. In
Psychiatrie, ein Lehrbuch für Studierende und Ärtze (English transl. R. M. Barclay, 1919). E. \& S. Livingstone: Edinburgh.

Manschreck, T. C., Maher, B. A., Rucklos, M. E. \& Vereen, D. R. (1982). Disturbed voluntary motor activity in schizophrenic disorder. Psychological Medicine 12, 73-84.

Marsden, C. D., Tarsy, D. \& Baldessarini, R. J. (1975). Spontaneous and drug-induced movement disorder in psychiatric patients. In Psychiatric Aspects of Neurological Disease (ed. D. F. Benson and D. Blumer), pp. 219-226. Grune \& Stratton: New York. 